\title{
EFFECT OF DIFFERENT SUBSTRATES AND NUTRIENT SOLUTIONS ON VEGETATIVE GROWTH, MINERAL CONTENT, PRODUCTION AND FRUIT QUALITY OF STRAWBERRY
}

\author{
Sayed F. El-Sayed ${ }^{1 *}$, H.A. Hassan ${ }^{1}$, M. Abul-Soud ${ }^{2}$ and Doaa A. M. Gad ${ }^{2}$ \\ 1. Veg. Crops Dept., Fac. Agric., Cairo Univ., Giza, Egypt \\ 2. Cent. Lab. Agric. Climate, Agric. Res. Cent., Dokki, Giza, Egypt
}

\begin{abstract}
The impact of three substrate mixtures and three nutrient solutions on the vegetative growth, mineral content, production and fruit quality of strawberry (Fragaria $\times$ ananassa, cv. Festival) was studied under unheated double-span plastic house conditions, at the Central Laboratory for Agricultural Climate (CLAC), Agricultural Research Center (ARC) during the two winter seasons of 2012/2013 and 2013/2014. The three substrate mixtures were perlite:peat-moss (1:1 V/V) M1, perlite: plant compost $(4: 1 \mathrm{~V} / \mathrm{V}) \mathrm{M} 2$, and perlite : vermicompost $(4: 1 \mathrm{~V} / \mathrm{V}) \mathrm{M} 3$, while the three nutrient solutions were vermicompost-tea, animal compost-tea and mineral nutrition (control). The tested factors were arranged in factorial design with three replicates. Obtained results indicated that all studied characteristics of the vegetative growth, yield and its components, fruit quality and chemical characteristics were greater by using the substrates mixture perlite: peat-moss as compared to the other two mixtures, whereas firmness, fruit acidity (TA) and vitamins $C$ in fruits were high by using substrate mixtures perlite: vermicompost. The highest concentration of heavy metals $(\mathrm{Ni}$ and $\mathrm{Pb})$ in fruits was detected when using substrate mixture perlite: plant compost was used. Regarding effect of nutrient solution, the mineral fertilizer (control) significantly increased vegetative growth, yield and its components, TSS, fruit taste, vitamins C and chemical characteristics compared to other tested nutrient solutions. However, the fruit firmness, TA and heavy metals were significantly higher when animal compost tea was used. The highest significant values of vegetative growth, yield and its component, fruit quality and chemical characteristics were recorded for plants grown in perlite: peat-moss mixture and fertigated with the mineral nutrient (control), while plants grown in perlite: vermicompost mixture and got animal compost-tea as nutrient showed the highest values of fruit firmness, TA and heavy metals.
\end{abstract}

Key words: Strawberry (Fragaria $\times$ ananassa), growth, yield, quality, chemical characteristics, substrate mixtures, vermicompost tea, animal compost, A-shape, soilless culture.

\section{INTRODUCTION}

Strawberry (Fragaria $\times$ ananassa Duch.) is one of the most important vegetable crops grown in Egypt for fresh consumption, processing and exportation. It's the unique vegetable crop belong to family Rosaceae. Soil pests (soil borne diseases, nematodes and weeds) are one of the most important limiting factors that have

* Corresponding author: Tel. : +201004987480

E-mail address: sayedfathey2000@yahoo.com negative influence on vegetable yield and quality (Carpenter et al., 2000).

Soilless strawberry culture using greenhouses extends the crop period, allows out of season production and increases the yield. Moreover, hydroponic production has been used for thousands of years where suitable soil is unavailable for growth or where the soil is contaminated (Jafarnia et al., 2010), where 
growth media and nutrition are the most important factors in hydroponic production (Hesami et al., 2012).

Concerning growth media, at the beginning gravel or sand, later materials such as peat, vermiculite, perlite have been used commonly (Celikel, 1999). Nowadays, using mixture of peat moss and perlite is one of the mostly used substrate for production of hydroponic strawberries in developing countries (Jafarnia et al., 2010). Peat moss is the most wide use substrate for seedlings production and commercial vegetable production (Robertson, 1993; Abul-Soud et al., 2015). Its long-time success is certainly due to the physical properties (slow degradation rate, low bulk density, high porosity, high water holding capacity) and the chemical characteristics (relatively high cation exchange capacity, CEC) that makes peat particularly suitable as growing media for a large number of vegetables and ornamentals (Bohlin and Holmberg, 2004; Fascella, 2015). However, the environmental and ecological concerns in the recent years led to minimize the use of peat because its harvest is destroying endangered wetland ecosystems worldwide (Robertson, 1993). Another reason for the search of a peat substitute included the high price, especially in countries without peat moss resources and environmental constraints (Abad et al., 2001). One the other hand, due to high price and not easy availability of peat in Egypt, it is need to produce local substrate instead of imported substrates. Moreover, for optimizing the soilless culture inputs and maximizes the production with concerns on the environmental impacts, it is need to develop the ecology soilless culture system via alternating the peat moss with compost and vermicompost and replace the chemical nutrient solution by organic sources of nutrient solution.

Compost and vermicompost are two organic sources in Egypt which may play the role of peat moss without any negative effects on a variety of crops raised in these substrates. Compost is the material that results when recycled plant wastes, biosolids (solid materials like manure), fish, and other organic materials decompose aerobically through the action of microorganisms that live in the presence of air.
Depending on the organic matter being composted, it may take up to six months to produce a mature batch of compost (Baldwin and Greenfield, 2000). One the other hand, vermicompost is the product of accelerated biodegradation of organic matter through the use of high densities of earthworms without a thermophilic stage (Dominguez et al., 1997). Vermicompost is fine textured peat-like organic material with high porosity, aeration, drainage, water-holding capacities, and low $\mathrm{C}: \mathrm{N}$ ratios (Domínguez, 2004). Different previous studies revealed that peat can be substituted with compost (Mahmoud et al., 2014) and vermicompost (Hashemimajd et al., 2004), negative effects on lettuce and tomato, respectively. Furthermore, the partial substitution of the substrate mixture (perlite + cocopeat) with vemicompost (Ameri et al., 2012a ; Bidaki et al., 2013) or cattle manure (Bidaki et al., 2013) caused a remarkable improvements for strawberry yield (Ameri et al., 2012b; Bidaki et al., 2013) and average fruit weight (Ameri et al., 2012b).The effect of different vermicompost rates mixed with the standard substrate peat moss: perlite on vegetative growth and yield of sweet pepper (Capsicum annuum L. cv. Reda) grown in pots culture was studied by Abol-Soud et al. (2014). They used perlite: peatmoss: vermicompost (45:45:10) (Mix.10\%), perlite: peat moss: vermicompost (40:40:20) (Mix.20\%), perlite: peat moss: vermicompost $(35: 35: 30)$ (Mix. 30\%) and perlite: peat moss (50:50 V/V) (Control). The best vegetative growth and yield of sweet pepper were given by Mix.20\% vermicompost mixture followed by Mix. 10\% and Mix.30\% vermicompost mixture. In another study, it was found that the use of sand: vermicompost $(80: 20)$ recorded higher vegetative, yield characteristics and N, P and K contents of lettuce, celery, salad and red cabbage compared to peat moss: perlite (50:50) (Abul-Soud et al., 2015).

Compost tea is the commercially and anecdotally popularized term for an infusion where compost is steeped in water for a period of time with the aim of transferring soluble organic matter, beneficial micro-organisms and macro- and micro-nutrients into solution (NOSB, 2004). Vermicompost tea is watery extracts of vermicomposted materials made for 
their beneficial effects on plants (Litterick et al., 2004). Compost tea and vermicompost tea are made from compost and vermicompost, respectively, in a commercial tea brewer. Little work has been done to assess the nutritional benefits of compost tea on plant growth. Nonaerated compost tea has been reported to be as effective as inorganic fertilizer in promoting growth of strawberry plants (Hargreaves et al., 2009). Furthermore, strawberry yield was increased with the application of an aerated compost tea compared to a control solution (Welke, 2005). Vermicompost tea also increased plant production and mineral nutrient content (N, P, K, Ca and Mg) in pakchoi (Brassica rapa Cv. Bonsai Chinesis group) as compared to mineral solution (Pant, 2009; Pant et al., 2009). However, Arancon et al. (2007) reported that there were significant differences between aerated and non-aerated vermicompost tea in their effect, where aerated vermicompost tea had a more positive impact on plant growth than non-aerated tea extracted for the same period of time (24 hr.). Abul-Soud et al., (2015) revealed that chemical nutrient solution recorded higher values of vegetative growth traits, characteristics of yield (early and total) and fruits [average weight, vitamin C, firmness and TSS (\%)] and $\mathrm{N}, \mathrm{P}$ and $\mathrm{K}$ contents of leaves, lower fruit acidity as compared to compost tea and vermicompost tea. The objective of this study was to get on the highest vegetative growth, yield and quality characteristics for strawberry plants by using best substrate mixtures or nutrient solutions or together under A-shape technique system.

\section{MATERIALS AND METHODS}

This study was carried out in the experimental station of Central Laboratory for Agricultural Climate (CLAC), Agriculture Research Center (ARC), Egypt, throughout the two winter seasons of 2012/2013 and 2013/2014 under unheated double-span plastic house conditions in closed systems of soilless culture (substrate cultures) in A-shape.

\section{Plant Material}

Fresh transplants of strawberry cv. Festival were used in both seasons of the study. Fresh transplants were obtained from Strawberry Improvement Center, Faculty of Agric., Ain Shams University, Shoubra El-Kheima. Fresh transplants with three to four leaves were transplanted. Seedlings were dipped in Rhizolex solution at rate of 2 gram per liter before transplanting. Strawberry transplants were cultivated on $1^{\text {st }}$ week of October 2012 and 2013. They were transplanted directly into PVC pipes (4 inch). The final plant spacing was 25 $\mathrm{cm}$.

\section{Experimental Materials}

A metal A- shape system of soilless culture was used to present the substrate systems. The metal A- shape frame $(0.9$ m width and $1.3 \mathrm{~m}$ height) included three levels of PVC pipes (4 inch) on two sides. The PVC pipes were holed every $25 \mathrm{~cm}$. The A-shape performed plant density of 24 plants per square meter. The PVC pipes filled with the different studied substrates for illustrating the substrate culture system. The cultivated strawberry plants were irrigated by using drippers of 4 liter per hour capacity.

This system had a sloping 1\%. Different nutrient solutions were pumped via submersible pump (110 watt). The drainage was collected back to the tanks of the different systems in the close type of soilless culture. The fertigation rate was programmed to be 8 times / day.

\section{Experimental Treatments}

The experiment included two factors, namely three growth media which were substrate mixtures of perlite + peat moss (1:1 V/V) (M1), perlite + vermicompost (4:1 V/V) (M2) and perlite + plant compost $(4: 1 \mathrm{~V} / \mathrm{V})(\mathrm{M} 3)$, three sources of nutrient solutions (vermicompost-tea, animal compost-tea and chemical nutrient solution of El-Behairy (1994) as a (control). The EC level of all nutrient solutions was adjusted at 1.5 mmhos $^{-1}$ during vegetative stage under unheated plastic house condition. The experimental was factorial design with 3 replicates. Each experimental plot contained 10 plants. The sources of fertigation were assigned as main plots and substrate mixtures as subplots. 


\section{The substrates}

The physical and chemical properties of the different substrate mixtures that were used in average two seasons are illustrated in Table 1. Bulk density (BD), total pore space (TPS), water hold capacity (WHC) and air porosity (AP) were determined according to Wilson, (1983) and Raul, (1996). The $\mathrm{pH}$ of the potting mixtures were determined using a double distilled water suspension of each potting mixture in the ratio of 1:10 (W:V) (Inbar et al., 1993) that was agitated mechanically for 2 hrs and filtered through Whatman No.1 filter paper. The same solution was measured for electrical conductivity (EC mmhos ${ }^{-1}$ ) with a conductance meter that was standardized with 0.01 and $0.1 \mathrm{M} \mathrm{KCl}$.

\section{Preparing of the nutrition tea}

Two sources of nutrition tea (vermi and animal compost-tea) were prepared by soaking 6 $\mathrm{kg}$ of each compost in water tank (120 liters) to get the concentrated extractions that were used as nutrient solutions after dilution. Filtration was made before using the (animal and vermi) compost-tea to get the clear solution for fertilizing strawberry and to prevent the dust included in the drippers.

The animal compost was from cattle farm at Nubaria, the plant compost was from El Neil co. and vermicompost was from Central Laboratory for Agricultural Climate "Integrated environmental management of urban organic wastes using vermicomposting project".

The EC of the different nutrient solutions were adjusted by using EC meter to the required level (1.5 mmhos $\left.{ }^{-1}\right)$ during the plant growth and harvesting. The chemical compositions of vermicompost-tea, animal compost-tea and chemical nutrient solution were illustrated in Table 2.

\section{Data Recorded}

Samples of three plants were taken from each experimental plot to determine growth parameters (180 days after the transplanting date) mid of the two seasons as follows: plant height, number of leaves/plant, shoot fresh weigh, shoot dry weight and leaf chlorophyll reading by using Minolta Chlorophyll Meter
SPAD-501. In addition, samples of five plants of each experimental plot were labeled to determine fruit parameters as follows: early yield (determined as weight of all harvested fruits during November, December and January), total yield (calculated as the fresh weight of all harvested fruits all over the growing season (from $30^{\text {th }}$ November until $28^{\text {th }}$ May), average fruit weight and number of fruits/plant. On 30 March, for determination of fruit quality characteristics a random sample of 10 fruits was taken at full ripe stage from each experimental plot where, vitamin $\mathrm{C}$ in the fresh fruits (by using the 2, 6 Dichlorophenolindophenol method), firmness (by using Penetrometer (Fruit Pressure Tester) mod. FT 327), total soluble solids percentage (using a hand refractometer), titratable acidity (by titration with $0.1 \mathrm{~N}$ of $\mathrm{NaOH}$ solution, using phenolphthalein indicator) and fruit taste (calculated as ratio of the total soluble solids content to total acidity), were determined according to the method described in AOAC (1990). Moreover, $100 \mathrm{~g}$ fresh samples of leaves and fruits were oven dried at $70^{\circ} \mathrm{C}$ for $3 \mathrm{hr}$., then ground in a blender and were digested using sulfuric acid and hydrogen peroxide as described by (Chapman and Pratt, 1961). Thereafter, $\mathrm{N}, \mathrm{P}$ and $\mathrm{K}$ were determined in leaves and fruit samples. Micro-Kjeldahle method was used for $\mathrm{N}$ determination, while colorimetric method were used for $\mathrm{P}$ and $\mathrm{K}$ determination using spectrophotometer and flame photometer, respectively, as was described by Chapman and Pratt (1961). In addition, $\mathrm{Fe}, \mathrm{Mn}, \mathrm{Cu}, \mathrm{Zn}$ and $\mathrm{Cd}, \mathrm{Pb}$, and $\mathrm{Ni}$ were determined spectrometrically in fruits using Phillips Unicum Atomic Absorption Spectrometer as was described by Chapman and Pratt (1961).

\section{Statistical Analysis}

SAS program was used for statistical analysis. The differences among means for all traits were tested for significance at 5\% level according to the procedure described by Snedecor and Cochran, (1981). 
Table 1. Physical and chemical properties of the different substrate mixtures in the two studies

\begin{tabular}{lccccccc}
\hline \multicolumn{1}{c}{ Physical } & & & & \multicolumn{3}{c}{ Chemical } \\
\hline Substrate & BD & TPS & WHC & AP & EC & pH \\
& $\mathbf{( k g / l )}$ & $\mathbf{( \% )}$ & $\mathbf{( \% )}$ & $\mathbf{( \% )}$ & $\left.(\mathbf{m m h o s})^{-1}\right)$ & \\
\hline Perlite: Peat moss (1:1(V/V)) (M1) & 0.140 & 65.25 & 52.8 & 12.5 & 0.20 & 5.0 \\
Perlite: Vermicompost (4:1(V/V)) (M2) & 0.277 & 62.87 & 43.34 & 19.53 & 1.7 & 7.2 \\
Perlite: Plant compost (4:1(V/V)) (M3) & 0.275 & 60.92 & 44.14 & 16.78 & 4.84 & 8.4 \\
\hline
\end{tabular}

*Bulk density= BD (kg/l), total pore space= TPS (\%), water hold capacity= WHC (\%) and air porosity= AP (\%)

Table 2. Concentrations of the different nutrient solutions (ppm)

\begin{tabular}{lccc}
\hline Element & Mineral & Vermi-compost tea & Animal compost tea \\
\hline $\mathbf{N}$ & 135 & 110.13 & 158.63 \\
$\mathbf{P}$ & 33.75 & 15.80 & 17.44 \\
$\mathbf{K}$ & 225 & 152.56 & 94.29 \\
$\mathbf{C a}$ & 135 & 100.61 & 72.86 \\
$\mathbf{M g}$ & 45 & 50.38 & 30 \\
$\mathbf{F e}$ & 2.7 & 10.07 & 7.50 \\
$\mathbf{M n}$ & 0.75 & 0.60 & 0.83 \\
$\mathbf{C u}$ & 0.375 & 0.15 & 0.11 \\
$\mathbf{Z n}$ & 0.113 & 0.15 & 0.10 \\
$\mathbf{B}$ & 0.188 & 0.19 & 0.38 \\
$\mathbf{M o}$ & 0.009 & - & - \\
$\mathbf{E c}$ & 1.5 & 1.5 & 1.5 \\
$\mathbf{p H}$ & 7.2 & 7.8 & 7.5 \\
\hline
\end{tabular}

\section{RESULTS}

\section{Vegetative Growth Characteristics}

Influence of substrate mixtures, nutrient solutions and their interaction on the number of leaves per plant, plant height, leaf chlorophyll reading (SPAD) unites and fresh and dry weights of shoots 180 (DAT) (at the midseason) are presented in Table 3 . The first season, using substrate mixture M1 (perlite: peat-moss $(1: 1 \mathrm{~V} / \mathrm{V})$ led to significant increase in the vegetative growth parameters as compared to the other two substrate mixtures M2 (perlite: vermicompost $(4: 1 \mathrm{~V} / \mathrm{V})$ and M3 (perlite: plant compost $(4: 1 \mathrm{~V} / \mathrm{V})$. In contrast, M2 mixture significantly produced the lowest values of vegetative growth characters. The same result was found in the second season.
Regarding nutrient solutions, mineral nutrient solution had a significant stimulation effect on the vegetative growth parameters compared with the other tested nutrient solutions. On the contrary, organic nutrition caused significant inhibition effect on all vegetative growth, and the worse one in this respect was recorded for animal compost-tea. These results were true in the two seasons.

The effect of interaction between substrate mixtures and nutrient solutions on vegetative growth characters was significant in both seasons. The highest values of vegetative growth characters were recorded for the substrate mixture M1 combined with mineral fertilizers. On the contrary, the lowest values of number of leaves, plant height, leaf chlorophyll reading and fresh and dry weights of shoots were found for M2 mixture that was fertilized with the animal compost-tea in the two growing seasons. 
Table 3. Effect of substrate mixtures and nutrient solutions on number of leaves, plant height, leaf chlorophyll reading (SPAD) and fresh and dry weight of shoots at 180 days after transplanting at the two mid-winter seasons of 2012/2013 and 2013/2014

\begin{tabular}{|c|c|c|c|c|c|c|c|c|}
\hline \multicolumn{5}{|c|}{$2012 / 2013$} & \multicolumn{4}{|c|}{$2013 / 2014$} \\
\hline \multirow{2}{*}{$\begin{array}{l}\text { Substrate } \\
\text { mixture }\end{array}$} & \multicolumn{8}{|c|}{ Nutrient solution } \\
\hline & $\begin{array}{c}\text { Vermi.- } \\
\text { tea }\end{array}$ & $\begin{array}{c}\text { Animal- } \\
\text { tea }\end{array}$ & Mineral & Mean & $\begin{array}{c}\text { Vermi.- } \\
\text { tea }\end{array}$ & $\begin{array}{c}\text { Animal- } \\
\text { tea }\end{array}$ & Mineral & Mean \\
\hline \multicolumn{9}{|c|}{ Number of leaves } \\
\hline M1 & $17.87 b$ & $14.28 c$ & $20.54 a$ & $17.56 \mathrm{~A}$ & $18.83 \mathrm{~b}$ & $14.51 \mathrm{c}$ & $20.67 a$ & $18.00 \mathrm{~A}$ \\
\hline M2 & $10.20 \mathrm{e}$ & $9.88 \mathrm{e}$ & $11.95 \mathrm{~d}$ & $10.68 \mathrm{C}$ & $9.75 f g$ & $9.00 \mathrm{~g}$ & $12.40 \mathrm{e}$ & $10.38 \mathrm{C}$ \\
\hline M3 & 10.00e & $12.00 \mathrm{~d}$ & $13.50 \mathrm{c}$ & 11.83B & $10.65 f$ & $13.40 \mathrm{~d}$ & 13.70cd & $12.58 \mathrm{~B}$ \\
\hline Mean & 12.69B & $12.05 \mathrm{C}$ & $15.33 \mathrm{~A}$ & & 13.08B & $12.30 \mathrm{C}$ & $15.59 \mathrm{~A}$ & \\
\hline \multicolumn{9}{|c|}{ Plant height (cm) } \\
\hline M1 & $15.96 a$ & 13.35b & $16.48 \mathrm{a}$ & $15.26 \mathrm{~A}$ & $16.00 \mathrm{~b}$ & $13.36 \mathrm{c}$ & $16.51 \mathrm{a}$ & $15.29 \mathrm{~A}$ \\
\hline M2 & $12.07 \mathrm{~cd}$ & 11.46d & $12.32 \mathrm{c}$ & $11.95 \mathrm{C}$ & $12.08 \mathrm{f}$ & $11.51 \mathrm{~g}$ & 12.33ef & $11.98 \mathrm{C}$ \\
\hline M3 & $12.42 \mathrm{c}$ & $12.70 \mathrm{bc}$ & $13.29 b$ & $12.80 \mathrm{~B}$ & 12.54de & $12.75 \mathrm{~d}$ & $13.34 c$ & $12.88 \mathrm{~B}$ \\
\hline Mean & 13.48B & $12.50 \mathrm{C}$ & $14.03 \mathrm{~A}$ & & 13.54B & $12.54 \mathrm{C}$ & $14.06 \mathrm{~A}$ & \\
\hline \multicolumn{9}{|c|}{ Leaf chlorophyll reading (SPAD) } \\
\hline M1 & $51.67 b$ & $47.92 \mathrm{c}$ & $53.97 a$ & 51.19A & $50.03 b$ & $46.08 c$ & $54.14 a$ & $50.08 \mathrm{~A}$ \\
\hline M2 & $29.43 \mathrm{~h}$ & $25.80 \mathrm{i}$ & $39.70 \mathrm{e}$ & 31.64C & $27.54 \mathrm{~h}$ & $22.04 \mathrm{i}$ & $38.08 \mathrm{f}$ & $29.22 \mathrm{C}$ \\
\hline M3 & $35.07 \mathrm{~g}$ & $38.32 \mathrm{f}$ & $43.02 d$ & $38.80 \mathrm{~B}$ & $36.50 \mathrm{~g}$ & $39.34 \mathrm{e}$ & $44.24 d$ & $40.03 \mathrm{~B}$ \\
\hline Mean & 38.72B & $37.35 \mathrm{C}$ & $45.56 \mathrm{~A}$ & & 38.02B & $35.82 \mathrm{C}$ & $45.49 \mathrm{~A}$ & \\
\hline \multicolumn{9}{|c|}{ Shoot fresh weight (g/plant) } \\
\hline M1 & $47.09 \mathrm{~b}$ & $30.35 c$ & $62.56 a$ & 46.67A & $60.69 b$ & $33.01 \mathrm{c}$ & $82.81 \mathrm{a}$ & $58.83 \mathrm{~A}$ \\
\hline M2 & $17.11 \mathrm{~g}$ & $12.53 \mathrm{~h}$ & 28.73d & 19.46C & $21.11 \mathrm{f}$ & $12.24 \mathrm{~g}$ & 26.61de & $19.99 \mathrm{C}$ \\
\hline M3 & 26.47e & $20.59 f$ & 28.87cd & 25.31B & 26.27de & 25.37e & $27.38 d$ & 26.34B \\
\hline Mean & $30.22 \mathrm{~B}$ & $21.16 \mathrm{C}$ & $40.05 \mathrm{~A}$ & & 36.02B & $23.54 \mathrm{C}$ & $45.60 \mathrm{~A}$ & \\
\hline \multicolumn{9}{|c|}{ Shoot dry weight (g/plant) } \\
\hline M1 & 13.63b & $9.66 \mathrm{c}$ & $16.67 \mathrm{a}$ & $13.32 \mathrm{~A}$ & $15.64 b$ & $10.46 \mathrm{c}$ & $19.12 \mathrm{a}$ & $15.07 \mathrm{~A}$ \\
\hline M2 & $6.81 \mathrm{e}$ & $4.89 \mathrm{f}$ & $8.58 \mathrm{~d}$ & $6.76 \mathrm{C}$ & $7.23 \mathrm{f}$ & $4.94 \mathrm{~g}$ & $9.50 \mathrm{~cd}$ & $7.22 \mathrm{C}$ \\
\hline M3 & 7.43e & 7.37e & $8.75 \mathrm{~cd}$ & $7.85 \mathrm{~B}$ & 8.92de & 8.00ef & $9.57 \mathrm{~cd}$ & 8.83B \\
\hline Mean & $9.29 \mathrm{~B}$ & 7.31C & $11.33 \mathrm{~A}$ & & 10.59B & $7.80 \mathrm{C}$ & $12.73 \mathrm{~A}$ & \\
\hline
\end{tabular}

M1 = perlite: peat moss (1:1(V/V)), M2 = perlite: vermicompost (4:1(V/V)), M3 = perlite: plant compost (4:1 $(V / V))$, vermi.-tea $=$ vermicompost tea, animal-tea $=$ animal compost tea.

*Different letters indicate significant difference at $5 \%$. 
Meanwhile, the lowest value of number of leaves per plant was registered for M2 substrate combined with using the vermi-tea as a nutrient.

\section{Mineral (N, P and K) Contents of Leaves}

Data presented in Table 4 show clearly that there were significant differences among the tested organic substrates in total nitrogen, phosphorus and potassium in leaves, where the highest values of these elements were obtained by using the substrates mixture of M1. In contrast, the lowest values of the percentage of total nitrogen, phosphorus and potassium in leaves were recorded for substrates mixture of M2. These results were true and similar in the two seasons of study.

As shown in the same table, there were significant differences in the concentrations of $\mathrm{N}, \mathrm{P}$ and $\mathrm{K}$ in leaves among the different sources of nutrition. In this respect, fertigation of strawberry plants with the mineral nutrient solution, followed by vermi-tea, recorded significantly higher values of total nitrogen, phosphorus and potassium contents of leaves as compared to using animal compost- tea.

As for the interaction between the three substrates mixture and the three nutrient solutions it was clear that such interaction had significant effect on the percentages of total nitrogen, phosphorus and potassium in strawberry leaves. The highest values of total nitrogen, phosphorus and potassium concentrations in leaves were recorded in plants grown on substrates mixture consisting of M1 and fertigated with mineral nutrition solution, whereas the lowest values were recorded for strawberry plants cultured on the M2 substrate and received animal compost extract as a nutrient solution. These results were confirmed in both seasons.

\section{Fruits Yield}

Substrates, nutrient solutions and their interaction had a significant effect on early yield, total fruit yield, number of fruits and average fruit weight per plant (Table 5). The highest values of number of fruits, early and total yield and average fruit weight were obtained by using the substrate mixture that consisted of M1, while the lowest values in all studied fruit yield traits were obtained by using M2. These results were true and similar in the two seasons of study.

Strawberry plants fertigated with mineral nutrition solution significantly exceeded those fertigated with vermi and animal compost-tea in the early and total fruit yield and average fruit weight as well as number of fruits plant. Meanwhile, using vermicompost extract in nutrition of strawberry significantly produced higher yield characteristics as compared to plant feeded with animal compost-tea.

Regarding the interaction effect on yield characteristics, data revealed that the highest significant values were recorded for M1 substrate combined with fertigation using mineral nutrient, while the lowest significant values were obtained by using M2 substrate combined with animal compost-tea nutrition during the two seasons. In addition, using M1combined with vermi-tea and M3 combined with mineral gave the same positive effect on average fruit weight during both seasons of the study.

\section{Fruit Quality Characteristics}

The different applied substrate mixtures and nutrient solutions affected the strawberry fruit quality, i.e., TSS, firmness, total acidity (TA), fruit taste and vitamin C (Table 6). Using M2 mixture performed the highest values of firmness, total acidity (TA) and vitamin C, whereas the highest values of TSS and fruit taste were recorded for M1 mixture. On the contrary, using M1 mixture gave the lowest values of firmness, TA and vitamin $C$, while the lowest values of TSS and fruit taste were recorded for M2 mixture during the two seasons.

Concerning the effect of nutrient solution, using animal compost-tea showed significantly the highest values of firmness and TA. In addition, the TSS, fruit taste and vitamin $\mathrm{C}$ were raised by using mineral fertilizer. In contrast, the lowest values of firmness and TA were obtained by using mineral fertilizer, while using vermitea produced the lowest values of vitamin C. Also, fruit taste and TSS were negatively affected by using animal compost-tea during the two seasons of study. 
Table 4. Effect of substrate mixtures and nutrient solutions on macro nutrient concentration in the fourth leaf of strawberry plants at 180 days after transplanting in two mid-winter seasons of 2012/2013 and 2013/2014

\begin{tabular}{|c|c|c|c|c|c|c|c|c|}
\hline \multicolumn{5}{|c|}{$2012 / 2013$} & \multicolumn{4}{|c|}{$2013 / 2014$} \\
\hline \multirow{2}{*}{$\begin{array}{l}\text { Substrate } \\
\text { mixture }\end{array}$} & \multicolumn{8}{|c|}{ Nutrient solution } \\
\hline & $\begin{array}{c}\text { Vermi.- } \\
\text { tea }\end{array}$ & $\begin{array}{c}\text { Animal - } \\
\text { tea }\end{array}$ & Mineral & Mean & $\begin{array}{c}\text { Vermi.- } \\
\text { tea }\end{array}$ & $\underset{\text { tea }}{\text { Animal- }}$ & Mineral & Mean \\
\hline \multicolumn{9}{|c|}{ N (\%) } \\
\hline M1 & $2.78 b$ & $2.68 c$ & $2.89 a$ & $2.78 \mathrm{~A}$ & $2.57 \mathrm{~b}$ & $2.56 c$ & $2.81 \mathrm{a}$ & $2.64 \mathrm{~A}$ \\
\hline M2 & $2.19 g$ & $2.13 \mathrm{~h}$ & $2.62 d$ & $2.37 \mathrm{C}$ & $2.00 \mathrm{~h}$ & $1.71 \mathrm{i}$ & 2.37e & $2.02 \mathrm{C}$ \\
\hline M3 & $2.39 \mathrm{e}$ & $2.37 f$ & $2.68 c$ & $2.48 \mathrm{~B}$ & $2.32 \mathrm{f}$ & $2.04 \mathrm{~g}$ & $2.46 \mathrm{~d}$ & $2.27 \mathrm{~B}$ \\
\hline Mean & $2.45 \mathrm{~B}$ & $2.40 \mathrm{C}$ & $2.73 \mathrm{~A}$ & & $2.29 \mathrm{~B}$ & $2.10 \mathrm{C}$ & $2.54 \mathrm{~A}$ & \\
\hline \multicolumn{9}{|c|}{$\mathbf{P}(\%)$} \\
\hline M1 & $0.565 b$ & $0.535 c$ & $0.588 \mathrm{a}$ & $0.563 \mathrm{~A}$ & $0.660 \mathrm{~b}$ & $0.635 c$ & $0.675 a$ & $0.657 \mathrm{~A}$ \\
\hline M2 & $0.440 \mathrm{~h}$ & $0.431 \mathrm{i}$ & $0.504 \mathrm{e}$ & $0.458 \mathrm{C}$ & $0.460 \mathrm{~h}$ & $0.380 \mathrm{i}$ & $0.570 \mathrm{e}$ & $0.470 \mathrm{C}$ \\
\hline M3 & $0.475 \mathrm{~g}$ & $0.492 f$ & $0.518 \mathrm{~d}$ & $0.495 B$ & $0.500 \mathrm{f}$ & $0.465 g$ & $0.605 d$ & $0.523 \mathrm{~B}$ \\
\hline Mean & $0.493 B$ & $0.486 \mathrm{C}$ & $0.537 \mathrm{~A}$ & & $0.540 \mathrm{~B}$ & $0.493 \mathrm{C}$ & $0.617 \mathrm{~A}$ & \\
\hline \multicolumn{9}{|c|}{ K (\%) } \\
\hline M1 & $3.34 b$ & $3.10 c$ & $3.77 \mathrm{a}$ & $3.40 \mathrm{~A}$ & $3.28 b$ & 3.10c & $3.49 a$ & $3.29 \mathrm{~A}$ \\
\hline M2 & $2.83 g$ & $2.64 \mathrm{~h}$ & $2.90 \mathrm{~d}$ & $2.79 \mathrm{C}$ & $2.78 \mathrm{~g}$ & $2.62 \mathrm{~h}$ & $2.90 \mathrm{~d}$ & $2.77 \mathrm{C}$ \\
\hline M3 & $2.88 \mathrm{e}$ & $2.87 f$ & 3.10c & $2.95 \mathrm{~B}$ & $2.87 e$ & $2.83 \mathrm{f}$ & 3.10c & 2.93B \\
\hline Mean & 3.02B & $2.87 \mathrm{C}$ & $3.26 \mathrm{~A}$ & & $2.98 \mathrm{~B}$ & $2.85 \mathrm{C}$ & $3.16 \mathrm{~A}$ & \\
\hline
\end{tabular}

M1 = perlite: peat moss $(1: 1 \mathrm{~V} / \mathrm{V}), \mathrm{M} 2$ = perlite: vermicompost $(4: 1 \mathrm{~V} / \mathrm{V})$, M3 = perlite: plant compost (4:1 $V / V)$, vermi.-tea $=$ vermicompost tea, animal-tea $=$ animal compost tea.

*Different letters indicate significant difference at $5 \%$ 
Table 5. Effect of substrate mixtures and nutrient solutions on yield characteristics per plant in the two winter seasons of $2012 / 2013$ and $2013 / 2014$

\begin{tabular}{|c|c|c|c|c|c|c|c|}
\hline & $2012 / 2013$ & & & & $2013 /$ & 2014 & \\
\hline \multirow{2}{*}{$\begin{array}{l}\text { Substrate } \\
\text { mixture }\end{array}$} & \multicolumn{7}{|c|}{ Nutrient solution } \\
\hline & $\begin{array}{cc}\text { Vermi.- } & \text { Animal - } \\
\text { tea } & \text { tea }\end{array}$ & Mineral & Mean & $\begin{array}{l}\text { Vermi.- } \\
\text { tea }\end{array}$ & $\begin{array}{c}\text { Animal - } \\
\text { tea }\end{array}$ & Mineral & Mean \\
\hline
\end{tabular}

\begin{tabular}{|c|c|c|c|c|c|c|c|c|}
\hline \multicolumn{9}{|c|}{ Early yield (g/plant) } \\
\hline M1 & $107.95 b$ & 79.41c & $125.21 \mathrm{a}$ & 104.19A & $110.00 \mathrm{~b}$ & $80.97 \mathrm{c}$ & $128.27 \mathrm{a}$ & $106.41 \mathrm{~A}$ \\
\hline M2 & $60.98 \mathrm{~h}$ & $42.53 i$ & $64.71 \mathrm{~g}$ & 56.07C & $62.20 \mathrm{~g}$ & $43.14 \mathrm{~h}$ & $66.36 f$ & $57.23 \mathrm{C}$ \\
\hline M3 & $65.48 \mathrm{f}$ & 71.59e & $72.72 d$ & 69.93B & $66.64 f$ & $73.35 \mathrm{e}$ & 73.85d & $71.28 \mathrm{~B}$ \\
\hline Mean & 78.14B & $64.51 \mathrm{C}$ & $87.55 \mathrm{~A}$ & & 79.61B & $65.82 \mathrm{C}$ & $89.49 \mathrm{~A}$ & \\
\hline \multicolumn{9}{|c|}{ Total yield (g/plant) } \\
\hline M1 & $431.78 b$ & $317.64 c$ & 500.82a & $416.75 \mathrm{~A}$ & 439.99b & $323.88 c$ & 513.07a & $425.65 \mathrm{~A}$ \\
\hline M2 & 243.94h & $170.10 \mathrm{i}$ & $258.85 g$ & $224.30 \mathrm{C}$ & $248.79 \mathrm{~g}$ & $172.55 \mathrm{~h}$ & $265.43 f$ & $228.92 \mathrm{C}$ \\
\hline M3 & $261.92 f$ & $286.34 \mathrm{e}$ & $290.88 d$ & 279.71B & $266.54 f$ & 293.39e & $295.38 d$ & $285.11 B$ \\
\hline Mean & 312.55B & $258.03 C$ & $350.18 \mathrm{~A}$ & & 318.44B & $263.28 \mathrm{C}$ & $357.96 \mathrm{~A}$ & \\
\hline \multicolumn{9}{|c|}{ Number of fruits } \\
\hline M1 & $23.21 b$ & $17.73 \mathrm{~cd}$ & $27.01 \mathrm{a}$ & $22.65 \mathrm{~A}$ & $23.26 b$ & $17.77 \mathrm{c}$ & $27.11 \mathrm{a}$ & $22.71 \mathrm{~A}$ \\
\hline M2 & $14.82 \mathrm{f}$ & $12.16 \mathrm{~g}$ & $15.51 \mathrm{ef}$ & $14.16 \mathrm{C}$ & $15.63 \mathrm{e}$ & $12.84 \mathrm{f}$ & 16.06de & $14.84 \mathrm{C}$ \\
\hline M3 & 16.77de & $18.75 c$ & 15.71ef & 17.07B & 17.01cd & $17.75 \mathrm{c}$ & 16.31de & $17.03 \mathrm{~B}$ \\
\hline Mean & 18.27B & $16.21 \mathrm{C}$ & $19.41 \mathrm{~A}$ & & 18.64B & $16.12 \mathrm{C}$ & $19.83 \mathrm{~A}$ & \\
\hline \multicolumn{9}{|c|}{ Average fruit weight (g) } \\
\hline M1 & $18.62 a$ & 17.95ab & $18.56 a$ & 18.38A & $18.92 a$ & $18.26 \mathrm{a}$ & 18.93a & $18.70 \mathrm{~A}$ \\
\hline M2 & $16.49 \mathrm{~cd}$ & $14.03 \mathrm{e}$ & 16.73bc & $15.75 \mathrm{C}$ & $15.95 b$ & $13.50 \mathrm{c}$ & $16.55 \mathrm{~b}$ & $15.33 \mathrm{C}$ \\
\hline M3 & 15.66cd & $15.31 d$ & $18.54 a$ & 16.50B & $15.70 \mathrm{~b}$ & $16.54 \mathrm{~b}$ & $18.14 \mathrm{a}$ & 16.79B \\
\hline Mean & 16.92B & $15.76 \mathrm{C}$ & 17.94A & & 16.85B & $16.10 \mathrm{C}$ & 17.87A & \\
\hline
\end{tabular}

M1 = perlite: peat moss $(1: 1 \mathrm{~V} / \mathrm{V}), \mathrm{M} 2=$ perlite: vermicompost $(4: 1 \mathrm{~V} / \mathrm{V}), \mathrm{M} 3$ = perlite: plant compost $(4: 1 \mathrm{~V} / \mathrm{V})$, vermi.-tea $=$ vermicompost tea, animal-tea $=$ animal compost tea.

${ }^{*}$ Different letters indicate significant difference at $5 \%$ 
Table 6. Effect of substrate mixtures and nutrient solutions on quality characteristics of strawberry plants in two mid-winter seasons of 2012/2013 and 2013/2014

\begin{tabular}{|c|c|c|c|c|c|c|c|c|}
\hline \multicolumn{5}{|c|}{$2012 / 2013$} & \multicolumn{4}{|c|}{$2013 / 2014$} \\
\hline \multirow{2}{*}{$\begin{array}{l}\text { Substrate } \\
\text { mixture }\end{array}$} & \multicolumn{8}{|c|}{ Nutrient solution } \\
\hline & $\begin{array}{c}\text { Vermi.- } \\
\text { tea }\end{array}$ & $\begin{array}{c}\text { Animal - } \\
\text { tea }\end{array}$ & Mineral & $\overline{\text { Mean }}$ & $\begin{array}{c}\text { Vermi.- } \\
\text { tea }\end{array}$ & $\begin{array}{c}\text { Animal - } \\
\text { tea }\end{array}$ & Mineral & Mean \\
\hline \multicolumn{9}{|c|}{ TSS (\%) } \\
\hline M1 & $6.66 \mathrm{~b}$ & $5.88 \mathrm{~d}$ & $7.00 \mathrm{a}$ & $6.51 \mathrm{~A}$ & $6.78 c$ & $5.83 g$ & $7.15 \mathrm{a}$ & $6.59 \mathrm{~A}$ \\
\hline M2 & 5.87d & $5.16 \mathrm{~g}$ & $6.24 c$ & $5.76 \mathrm{C}$ & $6.30 \mathrm{e}$ & $5.63 \mathrm{~h}$ & $6.45 d$ & $6.12 \mathrm{C}$ \\
\hline M3 & $5.49 f$ & $5.80 \mathrm{e}$ & $6.69 b$ & 5.99B & $5.80 \mathrm{~g}$ & $6.00 \mathrm{f}$ & $7.00 \mathrm{~b}$ & $6.26 \mathrm{~B}$ \\
\hline Mean & $6.01 \mathrm{~B}$ & $5.61 \mathrm{C}$ & $6.65 \mathrm{~A}$ & & $6.29 \mathrm{~B}$ & $5.82 \mathrm{C}$ & $6.86 \mathrm{~A}$ & \\
\hline \multicolumn{9}{|c|}{ Firmness (mg/cm²) } \\
\hline M1 & $245.00 d$ & $260.00 \mathrm{~b}$ & $223.00 \mathrm{~g}$ & 242.67C & 231.25de & $240.63 b$ & $213.25 f$ & $228.38 \mathrm{C}$ \\
\hline M2 & $242.50 \mathrm{e}$ & $290.00 a$ & $245.00 d$ & $259.17 \mathrm{~A}$ & $230.63 e$ & 273.33a & $235.00 c$ & $246.32 \mathrm{~A}$ \\
\hline M3 & $255.00 c$ & $240.00 \mathrm{f}$ & $245.50 \mathrm{~d}$ & 246.83B & $241.25 b$ & 234.00c & $232.50 \mathrm{~d}$ & 235.92B \\
\hline Mean & 247.50B & 263.33A & $237.83 C$ & & 234.38B & 249.32A & 226.92C & \\
\hline \multicolumn{9}{|c|}{ Titratable acidity (\%) } \\
\hline M1 & $0.405 \mathrm{~h}$ & $0.416 g$ & $0.393 \mathrm{i}$ & $0.405 \mathrm{C}$ & $0.400 \mathrm{~h}$ & $0.413 \mathrm{~g}$ & $0.396 \mathrm{i}$ & $0.403 \mathrm{C}$ \\
\hline M2 & $0.506 \mathrm{~b}$ & $0.513 a$ & $0.448 d$ & $0.489 \mathrm{~A}$ & $0.508 \mathrm{~b}$ & $0.510 \mathrm{a}$ & $0.446 \mathrm{~d}$ & $0.488 \mathrm{~A}$ \\
\hline M3 & $0.423 \mathrm{f}$ & $0.452 c$ & $0.434 \mathrm{e}$ & $0.436 \mathrm{~B}$ & $0.426 f$ & $0.455 \mathrm{c}$ & $0.433 \mathrm{e}$ & $0.438 \mathrm{~B}$ \\
\hline Mean & $0.445 B$ & $0.460 \mathrm{~A}$ & $0.425 \mathrm{C}$ & & $0.445 B$ & $0.459 \mathrm{~A}$ & $0.425 \mathrm{C}$ & \\
\hline \multicolumn{9}{|c|}{ TSS/TA } \\
\hline M1 & $16.44 \mathrm{~b}$ & $14.13 d$ & $17.81 \mathrm{a}$ & 16.13A & $16.95 b$ & $14.12 \mathrm{e}$ & $18.05 a$ & 16.37A \\
\hline M2 & $11.61 \mathrm{~h}$ & $10.06 \mathrm{i}$ & $13.94 \mathrm{e}$ & 11.87C & $12.40 \mathrm{~h}$ & $11.03 \mathrm{i}$ & $14.45 \mathrm{~d}$ & $12.63 \mathrm{C}$ \\
\hline M3 & $12.99 f$ & 12.82g & $15.42 \mathrm{c}$ & 13.74B & $13.61 \mathrm{f}$ & $13.18 \mathrm{~g}$ & $16.16 \mathrm{c}$ & 14.32B \\
\hline Mean & $30.22 \mathrm{~B}$ & $21.16 \mathrm{C}$ & $40.05 \mathrm{~A}$ & & 36.02B & $23.54 \mathrm{C}$ & $45.60 \mathrm{~A}$ & \\
\hline \multicolumn{9}{|c|}{ V. C (mg/100g fw) } \\
\hline M1 & $66.05 \mathrm{e}$ & $65.34 \mathrm{e}$ & 72.33c & $67.90 \mathrm{C}$ & $65.15 f$ & $62.12 \mathrm{~g}$ & 72.53d & $66.60 \mathrm{C}$ \\
\hline M2 & $81.46 b$ & $82.15 b$ & $87.21 \mathrm{a}$ & 83.61A & $81.12 b$ & $80.71 b$ & 86.03a & $82.62 \mathrm{~A}$ \\
\hline M3 & 70.19d & 73.31c & 73.13c & 72.21B & $68.79 \mathrm{e}$ & $75.41 \mathrm{c}$ & $75.55 c$ & 73.25B \\
\hline Mean & $72.56 \mathrm{C}$ & 73.60B & $77.56 \mathrm{~A}$ & & 71.69C & $72.75 B$ & 78.04A & \\
\hline
\end{tabular}

M1 = perlite: peat moss (1:1 V/V), M2 = perlite: vermicompost (4:1(V/V), M3 = perlite: plant compost (4:1 V/V), vermi.-tea $=$ vermicompost tea, animal-tea $=$ animal compost tea.

*Different letters indicate significant difference at 5\% 
Concerning the effect of the interaction on quality characteristics of strawberry fruits, data in the same table revealed that using M1mixture combined with mineral nutrition gave the highest values of TSS and fruit taste. Meanwhile, the highest value of firmness and TA were found in plants grown on M2 mixture and fertigated with animal compost-tea. However, using M2 mixture combined with mineral nutrition showed the highest value of vitamin C. By contrast, the lowest values of TSS and fruit taste were found in the interaction between the mixture of M2 combined with animal compost-tea. In addition, using the combination between M1combined with mineral nutrition decreased the firmness and TA. Also, vitamin $\mathrm{C}$ was significantly and negatively affected by using M1 combined with animal compost-tea. It is notable that these results were confirmed during the two studied seasons.

\section{Nutrient Concentration in Strawberry Fruits}

\section{Macro and microelements}

The effect of growing substrates, nutrient solutions and their interaction on the concentration of some macro and microelements in strawberry fruits is shown in Tables 7 and 8. Generally, the highest percentages of nitrogen, phosphorus, potassium, iron, manganese, copper and zinc in strawberry fruits were recorded in plants grown on M1 mixture, whereas the mixture of M2 showed a reverse trend.

Comparing with mineral nutrition, fertigation of strawberry plants with vermi or animal compost-tea, significantly increased the concentrations of all determined elements (nitrogen, phosphorus, potassium, iron, manganese, copper and zinc) in strawberry fruits. The lowest concentrations of these elements were detected in fruits picked from plants fertigated with animal compost-tea.

The interaction effect between growing substrates and nutrient solutions revealed that fruits picked from plants grown on M1 mixture and fertigated with mineral nutrition contained the highest percentages of macro and microelements, whereas fruits of plants grown on the mixture M2 and fertigated with animal compost-tea contained the lowest concentrations of these elements. These results were true in both seasons.

\section{Heavy metals}

Data presented in Table 8 indicated that adding compost, whatever its source, i.e., vermi or plant compost, to perlite led to significant increase in the lead and nickel contents of fruits as compared with M1.

The effect of nutrient solutions on fruit contents of lead and nickel is significant. Fertigation of strawberry plants with compost tea (vermi and animal) caused significant increase in lead and nickel in strawberry fruits as compared with the mineral nutrient solution. The lowest lead and nickel content of fruits in this respect was found in fruits obtained from plants fertigated with mineral nutrient solution.

The effect of the interaction between growing substrates and nutrient solutions on heavy metals concentrations in strawberry fruits was significant. The highest concentration of lead and nickel in strawberry fruits were recorded in fruits obtained from plant grown on substrate consisted of M2 combined with animal compost-tea fertigation. In contrast, the lowest lead and nickel concentrations were found in fruits picked from plants grown on M1 mixture and supplied with mineral nutrition.

Meanwhile, the study results showed that all treatments did not have a significant effect on cadmium content in fruits during the two seasons.

\section{DISCUSSION}

One of the success factors in hydrophonic system is substrate and nutrient solution management (Firoozabadi et al., 2009; PorHossein et al., 2009; Ebrahimi et al., 2012). The properties of different materials used as growing media exhibit direct and indirect effects on plant growth and productivity.The substrate, a medium in which roots can grow, also protected plant as physical support. It can be constituted of pure materials or mixtures. This is because this capacity leads to higher capacity of nutrients and better water management. Some technical and economic factors play role when 
Table 7. Effect of substrate mixtures and nutrient solutions on macronutrient concentration of strawberry fruits at 180 days after transplanting in two mid-winter seasons of 2012/ 2013 and 2013/2014

\begin{tabular}{|c|c|c|c|c|c|c|}
\hline \multicolumn{3}{|c|}{$2012 / 2013$} & \multicolumn{4}{|c|}{$2013 / 2014$} \\
\hline \multirow{2}{*}{$\begin{array}{l}\text { Substrate } \\
\text { mixture }\end{array}$} & \multicolumn{6}{|c|}{ Nutrient solution } \\
\hline & $\begin{array}{c}\text { Vermi.-teaAnimal- Mineral } \\
\text { tea }\end{array}$ & Mean & $\begin{array}{l}\text { Vermi.- } \\
\text { tea }\end{array}$ & $\underset{\text { tea }}{\text { Animal- }}$ & Mineral & Mean \\
\hline
\end{tabular}

\section{Nitrogen content in fruits (\%)}

$\begin{array}{lrrrrrrrr}\text { M1 } & 2.20 \mathrm{~b} & 2.04 \mathrm{~d} & 2.25 \mathrm{a} & 2.16 \mathrm{~A} & 2.19 \mathrm{~b} & 2.00 \mathrm{c} & 2.67 \mathrm{a} & 2.29 \mathrm{~A} \\ \text { M2 } & 1.74 \mathrm{f} & 1.44 \mathrm{~g} & 2.14 \mathrm{c} & 1.77 \mathrm{C} & 1.52 \mathrm{f} & 1.13 \mathrm{~g} & 1.84 \mathrm{~d} & 1.50 \mathrm{C} \\ \text { M3 } & 1.90 \mathrm{e} & 1.79 \mathrm{f} & 2.15 \mathrm{bc} & 1.95 \mathrm{~B} & 1.75 \mathrm{e} & 1.54 \mathrm{f} & 2.04 \mathrm{c} & 1.78 \mathrm{~B} \\ & & & & & & & & \\ \text { Mean } & 1.95 \mathrm{~B} & 1.75 \mathrm{C} & 2.18 \mathrm{~A} & & 1.82 \mathrm{~B} & 1.56 \mathrm{C} & 2.18 \mathrm{~A} & \end{array}$

\section{Phosphorus content in fruits (\%)}

$\begin{array}{ccccccccc}\text { M1 } & \text { 0.937b } & 0.898 \mathrm{c} & 0.947 \mathrm{a} & 0.927 \mathrm{~A} & 0.928 \mathrm{~b} & 0.913 \mathrm{c} & 0.967 \mathrm{a} & 0.936 \mathrm{~A} \\ \text { M2 } & 0.824 \mathrm{f} & 0.768 \mathrm{~g} & 0.865 \mathrm{e} & 0.819 \mathrm{C} & 0.803 \mathrm{e} & 0.783 \mathrm{f} & 0.913 \mathrm{c} & 0.833 \mathrm{C} \\ \text { M3 } & 0.881 \mathrm{~d} & 0.825 \mathrm{f} & 0.901 \mathrm{c} & 0.869 \mathrm{~B} & 0.843 \mathrm{~d} & 0.805 \mathrm{e} & 0.917 \mathrm{c} & 0.855 \mathrm{~B} \\ & & & & & & & & \\ \text { Mean } & 0.881 \mathrm{~B} & 0.830 \mathrm{C} & 0.904 \mathrm{~A} & & 0.858 \mathrm{~B} & 0.834 \mathrm{C} & 0.932 \mathrm{~A} & \end{array}$

Potassium content in fruits (\%)

$\begin{array}{crrrrrrrr}\text { M1 } & 2.52 \mathrm{~b} & 2.22 \mathrm{e} & 2.69 \mathrm{a} & 2.48 \mathrm{~A} & 2.21 \mathrm{~b} & 2.12 \mathrm{c} & 2.26 \mathrm{a} & 2.19 \mathrm{~A} \\ \text { M2 } & 2.05 \mathrm{~h} & 1.94 \mathrm{i} & 2.24 \mathrm{~d} & 2.07 \mathrm{C} & 1.62 \mathrm{~h} & 1.36 \mathrm{i} & 1.92 \mathrm{e} & 1.63 \mathrm{C} \\ \text { M3 } & 2.11 \mathrm{f} & 2.10 \mathrm{~g} & 2.46 \mathrm{c} & 2.22 \mathrm{~B} & 1.83 \mathrm{f} & 1.68 \mathrm{~g} & 1.93 \mathrm{~d} & 1.81 \mathrm{~B} \\ & & & & & & & & \\ \text { Mean } & 2.23 \mathrm{~B} & 2.09 \mathrm{C} & 2.46 \mathrm{~A} & & 1.88 \mathrm{~B} & 1.72 \mathrm{C} & 2.03 \mathrm{~A} & \end{array}$

M1 = perlite: peat moss $(1: 1 \mathrm{~V} / \mathrm{V}), \mathrm{M} 2$ = perlite: vermicompost $(4: 1 \mathrm{~V} / \mathrm{V}), \mathrm{M} 3=$ perlite: plant compost $(4: 1$ $V / V)$, vermi.-tea $=$ vermicompost tea, animal-tea $=$ animal compost tea.

*Different letters indicate significant difference at $5 \%$ 
Table 8. Effect of substrate mixtures and nutrient solutions on micronutrient concentration and heavy metals of strawberry fruits at 180 days after transplanting in two mid- winter seasons of 2012/2013 and 2013/2014

\begin{tabular}{|c|c|c|c|c|c|c|c|c|}
\hline \multicolumn{5}{|c|}{$2012 / 2013$} & \multicolumn{4}{|c|}{$2013 / 2014$} \\
\hline \multirow{2}{*}{$\begin{array}{l}\text { Substrate } \\
\text { mixture }\end{array}$} & \multicolumn{8}{|c|}{ Nutrient solution } \\
\hline & $\begin{array}{c}\text { Vermi.- } \\
\text { tea }\end{array}$ & $\begin{array}{c}\text { Animal - } \\
\text { tea }\end{array}$ & Mineral & Mean & $\begin{array}{c}\text { Vermi.- } \\
\text { tea }\end{array}$ & $\begin{array}{c}\text { Animal - } \\
\text { tea }\end{array}$ & Mineral & Mean \\
\hline \multicolumn{9}{|c|}{ Iron content in fruits (ppm) } \\
\hline M1 & $388.71 b$ & 328.63 & 391.81a & 369.72A & $388.71 b$ & $328.63 c$ & 391.81a & $369.71 \mathrm{~A}$ \\
\hline M2 & $178.46 \mathrm{~h}$ & $133.04 \mathrm{i}$ & $247.00 \mathrm{f}$ & 186.17C & $178.46 \mathrm{~h}$ & $133.04 \mathrm{i}$ & $280.67 \mathrm{e}$ & 197.39C \\
\hline M3 & 280.67e & 180.92 & 311.33d & 257.64B & 231.33f & $180.92 \mathrm{~g}$ & 294.22d & $235.49 B$ \\
\hline Mean & 282.61B & 214.20C & $316.72 \mathrm{~A}$ & & 266.17B & 214.20C & $322.23 \mathrm{~A}$ & \\
\hline \multicolumn{9}{|c|}{ Manganese content in fruits (ppm) } \\
\hline M1 & $53.27 \mathrm{~b}$ & $48.67 \mathrm{c}$ & $55.23 a$ & 52.39A & $55.86 b$ & $51.33 c$ & $60.50 \mathrm{a}$ & $55.90 \mathrm{~A}$ \\
\hline M2 & $22.84 \mathrm{~g}$ & $15.58 \mathrm{~h}$ & 46.79d & $28.40 \mathrm{C}$ & $23.67 \mathrm{~g}$ & $15.67 \mathrm{~h}$ & $49.00 \mathrm{~d}$ & $29.45 \mathrm{C}$ \\
\hline M3 & $43.83 \mathrm{e}$ & $26.67 f$ & $48.29 c$ & 39.60B & $27.36 f$ & $34.67 \mathrm{e}$ & $51.35 c$ & 37.79B \\
\hline Mean & 39.98B & $30.30 \mathrm{C}$ & $50.10 \mathrm{~A}$ & & 35.63B & 33.89C & 53.61A & \\
\hline \multicolumn{9}{|c|}{ Copper content in fruits (ppm) } \\
\hline M1 & $66.29 b$ & $56.75 c$ & $74.97 a$ & $66.01 \mathrm{~A}$ & $67.00 \mathrm{~b}$ & $54.00 \mathrm{c}$ & 70.33a & $63.78 \mathrm{~A}$ \\
\hline M2 & $33.79 \mathrm{~g}$ & $24.42 \mathrm{~h}$ & $52.00 \mathrm{~d}$ & $36.74 \mathrm{C}$ & $30.67 f$ & 26.33g & $49.00 \mathrm{~d}$ & $35.33 \mathrm{C}$ \\
\hline M3 & $37.46 \mathrm{e}$ & $34.92 \mathrm{f}$ & $52.13 d$ & 41.50B & $35.00 \mathrm{e}$ & $31.33 \mathrm{f}$ & 53.00c & 39.78B \\
\hline Mean & 45.85B & $38.70 \mathrm{C}$ & $59.70 \mathrm{~A}$ & & 44.22B & $37.22 \mathrm{C}$ & 57.44A & \\
\hline \multicolumn{9}{|c|}{ Zinc content in fruits (ppm) } \\
\hline M1 & $20.03 b$ & $19.83 \mathrm{bc}$ & $24.43 a$ & $21.43 \mathrm{~A}$ & $21.67 b$ & $18.67 \mathrm{c}$ & $25.00 \mathrm{a}$ & $21.78 \mathrm{~A}$ \\
\hline M2 & $15.50 \mathrm{e}$ & $12.50 f$ & $18.75 c$ & 15.59C & $14.33 f$ & $12.67 \mathrm{~g}$ & 19.00c & $15.33 \mathrm{C}$ \\
\hline M3 & 17.13d & 16.00de & $19.75 b c$ & 17.63B & $17.00 \mathrm{~d}$ & $15.67 \mathrm{e}$ & 19.33c & 17.33B \\
\hline Mean & 17.55B & $16.11 \mathrm{C}$ & $20.98 \mathrm{~A}$ & & 17.67B & $15.67 \mathrm{C}$ & $21.11 \mathrm{~A}$ & \\
\hline \multicolumn{9}{|c|}{ Lead content in fruits (ppm) } \\
\hline M1 & $0.002 \mathrm{~g}$ & $0.037 \mathrm{f}$ & $0.001 \mathrm{~g}$ & $0.013 \mathrm{C}$ & $0.005 \mathrm{~h}$ & $0.039 f$ & $0.002 \mathrm{i}$ & $0.015 \mathrm{C}$ \\
\hline M2 & $0.045 \mathrm{~d}$ & $0.067 \mathrm{a}$ & $0.039 \mathrm{e}$ & $0.050 \mathrm{~B}$ & $0.048 \mathrm{~d}$ & $0.065 a$ & $0.030 \mathrm{~g}$ & 0.048B \\
\hline M3 & $0.056 \mathrm{c}$ & $0.062 b$ & $0.038 \mathrm{ef}$ & $0.052 \mathrm{~A}$ & $0.056 \mathrm{c}$ & $0.060 \mathrm{~b}$ & $0.042 \mathrm{e}$ & $0.053 \mathrm{~A}$ \\
\hline Mean & $0.034 \mathrm{~B}$ & $0.055 \mathrm{~A}$ & $0.026 \mathrm{C}$ & & $0.036 \mathrm{~B}$ & $0.055 \mathrm{~A}$ & $0.025 \mathrm{C}$ & \\
\hline \multicolumn{9}{|c|}{ Nickel content in fruits (ppm) } \\
\hline M1 & $0.036 f g$ & $0.037 \mathrm{f}$ & $0.035 \mathrm{~g}$ & $0.036 \mathrm{C}$ & 0.038gh & $0.039 \mathrm{~g}$ & $0.037 \mathrm{~h}$ & $0.038 \mathrm{C}$ \\
\hline M2 & $0.047 \mathrm{~d}$ & $0.068 \mathrm{a}$ & $0.044 \mathrm{e}$ & $0.053 \mathrm{~B}$ & $0.051 \mathrm{~d}$ & $0.071 \mathrm{a}$ & $0.046 f$ & $0.056 \mathrm{~B}$ \\
\hline M3 & $0.066 \mathrm{~b}$ & $0.056 c$ & $0.045 \mathrm{e}$ & 0.056A & $0.069 \mathrm{~b}$ & $0.060 \mathrm{c}$ & $0.048 \mathrm{e}$ & 0.059A \\
\hline Mean & $0.050 \mathrm{~B}$ & $0.054 \mathrm{~A}$ & $0.041 \mathrm{C}$ & & $0.053 B$ & $0.057 \mathrm{~A}$ & $0.044 \mathrm{C}$ & \\
\hline
\end{tabular}

M1 = perlite: peat moss $(1: 1 \mathrm{~V} / \mathrm{V}), \mathrm{M} 2$ = perlite: vermicompost $(4: 1 \mathrm{~V} / \mathrm{V})$, M3 = perlite: plant compost (4:1 $V / V)$, vermi.-tea $=$ vermicompost tea, animal-tea $=$ animal compost tea.

*Different letters indicate significant difference at $5 \%$ 
choosing substrates (Celikel, 1999 ; Ercisli et al., 2005). In the present study, compost and vermicompost were suggested as locally available and inexpensive soilless substrates with no pollution limitations and having adequate physical and chemical properties, instead of peat moss which is very expensive in Egypt, due to its importation from abroad. Compost is not only a safer beneficial product for the organic wastes which may cause severe air and/or water pollution (Raviv and Medina, 1997), but its integration in the growing media improves their fertilizing capacity. In addition, composts tend to have porosity and aeration properties comparable to those of peat and, as such are ideal substitutes in propagating media (Benito et al., 2005). Also, vermicompost is a sustainable solution for management of organic wastes which are major source of environmental pollution (Lazcano et al., 2009). It could be utilized in ecology soilless culture of different vegetables (Abul-Soud et al., 2014; AboSedera et al., 2015 ; Abul-Soud et al., 2015).

The present study proved that substitution peat moss with compost or vermicompost led to a significant deterioration in the vegetative growth characters (Table 4). Such significant deterioration may be attributed to the low concentration of total nitrogen, phosphorus and potassium in leaves that was determined during plant growth (Table 4). The low vegetative growth that happened due to using compost or vermicompost instead of peat moss led to lower number of fruits per plant and less average fruit weight which consequently caused a serious reduction in strawberry yield (Table 5). Vermicompost had more negative effect on the vegetative growth, nutrient uptake and yield than compost. The physical and chemical property of different substrate mixtures of both studies, revealed that both substrate mixtures (M3 and M2) had worse physical characters (BD, TPS, WHC and AP) than the standard substrates mixture (M1). The standard substrates mixture had lower bulk density, and air porosity and higher total pore space and water hold capacity than the other two mixtures. Meanwhile, alternating the standard mixture by adding vermicompost or compost to perlite instead of peat moss caused a serious increase in substrates mixture salinity from 0.2 to 1.7 and
$4.84 \mathrm{EC}$, respectively. The $\mathrm{pH}$ of the mixture was also raised from 5 in the case of using peat moss to 7.2 and 8.4 in the case of using vermicompost and compost, respectively (Table 1 ). Such deterioration in the physical characters and the mixture salinity may be responsible for the decrease in the N, P and K uptake. High salinity caused significant reduction in the vegetative growth and yield generally in different vegetable crops (Shannon and Grieve, 1999; Maksimovic and Ilin, 2002) and especially in strawberry (Pirlak and Esitken, 2004) which is very sensitive for salinity (Saied et al., 2005).

It has been shown that salinity reduces leaf number, leaf area, shoot dry weight and number of crowns and causes low strawberry yield (Pirlak and Esitken, 2004). Excess salt in the soil may adversely affect plant growth either through osmotic inhibition of water uptake by roots or specific ion effects. Specific ion effects may cause direct toxicity or alternatively, the insolubility or competitive absorption of ions may affect plant nutritional balances (Greenway and Munns, 1980). Salt stress has toxic effects on plants and lead to metabolic changes, like loss of chloroplast activity, decreased photosynthetic rate and increased photorespiration rate which then leads to an increased reactive oxygen species (ROS) production (Parida and Das, 2005). These results are in agreement with the present ones that revealed significant decrease in chlorophyll concentration in the leaves of plants grown in the present study in the mixtures of M3 and M2 that contained high salinity.

The main cause of reduced plant growth in the presence of salt can be impairment of water regime. Increasing the salt concentration in the soil increases the osmotic pressure of the soil solution and plants cannot uptake water as easily as in the case of relatively non-saline soils. Therefore, as the concentration of salt i.e., soil EC increases, water becomes less accessible to plants, even if the soil contains significant amounts of water and looks wet (Maksimovic and Ilin, 2002).

High concentrations of $\mathrm{NaCl}$ act antagonistically to the uptake of the other nutrients, such as $\mathrm{K}^{+}$, $\mathrm{Ca}_{2}{ }^{+}, \mathrm{N}, \mathrm{P}$ (Cramer et al., 1991; Grattan and Grieve, 1999). This may explain the low 
concentration of $\mathrm{N}, \mathrm{P}$ and $\mathrm{K}$ in strawberry leaves of plants grown in the present study in the mixtures of M3 and M2 that contained high salinity.

Some studies reported that vermicompost may decrease growth and even cause plant death (Roberts et al., 2007; Lazcano and Domínguez 2010). The variability in the effects of vermicompost may depend on the cultivation system into which it is incorporated, as well as on the physical, chemical and biological characteristics of vermicompost, which vary widely depending on the original feedstock, the earthworm species used, the production process, and the age of vermicompost (Rodda et al., 2006; Roberts et al., 2007). Alnayef (2012) revealed that salinity dramatically affected growth and yield in strawberry (Elsinore and Elsanta cultivars), although Elsinore appeared to be more impaired than Elsanta. Moreover a significant reduction of leaf photosynthesis, evaporation, and stomatal conductance was recorded 24 hrs., after salinity stress was applied in both cultivars

Adding compost or vermicompost to perlite led to reduction in fruit size and fruit contents of TSS, TSS/total acidity, N, P, K, iron, manganese, copper and zinc but meanwhile this addition increased fruit firmness, and fruit contents of acidity, vitamin $\mathrm{C}$ and heavy metals (lead and nickel). Again, the high salinity contents of compost and vermicompost mixtures might reduce the uptake and accumulation of $\mathrm{N}$, $\mathrm{P}$ and $\mathrm{K}$ in fruits. Accordingly, salt stressed fruit containing less sugar and more organic acids content have limited acceptance amongst consumers compared to the control (Keutgen and Pawelzik, 2007). TSS content varied between $7 \%$ and $12 \%$ depending on strawberry genotypes (Crespo, 2010). However, range of $7 \%$ minimum TSS content for acceptable quality of strawberry fruit (Mitcham et al., 2000). According to the results of Alnayef, (2012), TSS/TA was decreased under salinity conditions, which are in accordance with the present results.

Increasing salinity with $\mathrm{NaCl}$ reduced fruit size (Jensen, 2011; Alnayef, 2012), although there are differences in salinity tolerance between Fragaria $\times$ ananassa cultivars
(Keutgen and Pawelzik, 2008). The salinity treatments did not have any significant effect on fruit firmness (Jensen, 2011). On the other hand, the results of Alnayef (2012) revealed that fruit quality concerning fruit taste, aroma, appearance, TSS and TA, did not change but rather was enhanced under moderate salinity.

The low concentration of $\mathrm{N}, \mathrm{P}$ and $\mathrm{K}$ in strawberry fruits might be attributed to the high salinity of medium which act antagonistically to the uptake of the other nutrients, such as $\mathrm{K}^{+}$, $\mathrm{Ca}_{2}{ }^{+}$, N, P (Cramer et al., 1991; Grattan and Grieve, 1999). Also, increasing $\mathrm{pH}$ of the culture media, by adding compost and vermicompost may result in decreasing in the concentration of $\mathrm{Fe}$, $\mathrm{Mn}$ and $\mathrm{Zn}$ in fruits. These results agree with those of Ghehsareh and Samadi (2012) who indicated that in alkaline soils, high concentration of bicarbonate in soil solution increases soil $\mathrm{pH}$, decreases micronutrients concentration in soil solution and prevents the transfer of Fe, Zn, Mn, $\mathrm{Cu}$ and even $\mathrm{P}$ from root to stem and from stem to leaf.

The levels of heavy metals in the different composts are known to be much higher than in most agricultural soils (He et al., 1992), and depend on the origin of the compost. Pinamonti et al. (1997) reported that the use of compost from sewage sludge and poplar bark did not cause any significant increase in heavy metal levels in soil or plants in the short/medium term; by contrast, their experiments clearly demonstrated that the compost from municipal solid waste increased concentrations of $\mathrm{Zn}, \mathrm{Cu}, \mathrm{Ni}, \mathrm{Pb}$ and $\mathrm{Cr}$ in soil, and in the case of $\mathrm{Pb}$ and $\mathrm{Cd}$ also in the vegetation and the fruits. However, heavy metal levels were below the tolerated limits (600 $\mathrm{ppm}$ for $\mathrm{Pb}$ and $50 \mathrm{ppm}$ for $\mathrm{Ni}$ ) according to both the European and American regulations for soilless production of vegetables and ornamentals (Mazuela et al., 2012).

Regarding nutrient solutions, mineral nutrient solution had a significant simulative effect on the vegetative growth parameters compared with the other tested nutrient solutions. On the contrary, organic nutrition caused significant inhibition effect on all vegetative growth, and the worse one in this respect was recorded for animal compost-tea. 
Concerning nutrient solutions, plants fertigated with mineral solution showed more vigorous growth, higher number of fruits per plant, greater early and total yield, and their leaves were higher in $\mathrm{N}, \mathrm{P}$ and $\mathrm{K}$ when compared to the vermi and animal compost-tea treatments. The low N, P and $\mathrm{K}$ concentration in the plants fertilized with vermi or animal compost-tea may come from the low concentrations of these elements in these solution and which led to the week vegetative growth and consequently to the low strawberry yield. Similar results were reported by Jarecki et al. (2005) who carried out a study utilizing spent mushroom compost leachate and runoff compost leachate for plant growth in hydroponic culture as compared to Hoagland's hydroponic. Results from this study indicated that deficiency in nitrogen $(\mathrm{N})$ and phosphorus (P) on tomato and marigold plants were observed in both compost leachate treatments.

In the present study, tap water was added every three days to the organic solutions to keep the EC of these solutions at the 1.5 level. Adding water might reduce the nutrient element concentrations in these solutions. Similarly, Santiago-López et al. (2016) obtained lower yield, weight and size of organic produced cucumber compared to inorganically one and they mentioned that it could be attributed to the low nutrient concentration of organic fertilizer solutions. Gutiérrez-Miceli et al. (2008) revealed that low nutrient concentration in organic solutions resulted from the solutions dilution in order to avoid phytotoxicity in plants. The positive effect of the mineral nutrition on the vegetative growth and yield of strawberry in the present investigation may be also resulted from the balance among the compositions of the chemical nutrient solution that met the nutrient requirements of strawberry; such balance was absent among the elements of the animal compost and vermi-tea. In this respect, Márquez-Quiroz et al. (2014) reported that not only the low nutrient concentration but also the imbalanced nutrient ratios in the nutrient solutions affect the normal plant growth; thereby regularly result in a lower size and weight of fruits. Santiago-López et al. (2016) attributed the lower fruit weight and size in cucumber produced under the organic treatments mostly to the nitrogen deficit since it is known that an insufficient nitrogen supply during crop growth and development affects negatively vegetable and fruit yield, size and weight. Abol-Soud et al. (2015) also indicted that the concentrations and the balance among the elements of the mineral nutrition played a vital role in the highest results of vegetative, yield and quality characteristics as well as $\mathrm{N}, \mathrm{P}$ and $\mathrm{K}$ contents of strawberry leaves compared to the composition of vermi and animal compost-tea that had significant available amounts of plant nutrients but not in balance to meet strawberry requirements.

Moreover, strawberry fruits picked from plants received mineral nutrition were heavier in weight and had better nutritional quality (higher content of TSS and vitamin C and were higher in $\mathrm{N}, \mathrm{P}, \mathrm{K}, \mathrm{Mn}, \mathrm{Zn}, \mathrm{Cu}$ and $\mathrm{Fe}$ ). In contrast, these fruits were lower in fruits firmness, and had lower fruit contents of total acidity, lead and nickel as compared to those got organic nutrition. Higher total acidity is not a desirable property, where strawberry fruits with low total acidity and heavy metals (lead and nickel) are considered high quality fruits. In contrast to these results Santiago-López et al. (2016) recorded a higher antioxidant capacity for organic cucumber as compared to plants fertilized using inorganic nutrient solution. Herms and Mattson, (1992) stated that plants produced higher amounts of sugars (simple and complex) and secondary metabolites (pigments, vitamins, organic acids, terpenoides and phenolic compounds) when subjected to a deficit of soluble nitrogen supply. In general, there are many factors affecting compost-tea quality that may responsible for the contradiction in the results. These factors include added materials, aeration, brewing time, compost source and quality, extraction and mixing, foam, maintaining compost activity, mesh size of the tea bag or final filtration material, microbes in tea, ratio of compost to water and temperature (Ingham, 2005).

\section{Conclusion}

Using chemical nutrient solution with M1 medium gave the highest results of vegetative, yield and quality characteristics as well as N, P and $\mathrm{K}$ contents of strawberry leaves. Therefore, more research is required to enhance using vermicompost and plant compost as substrates and nutrient tea for production of strawberry hydroponically. 


\section{REFERENCES}

AOAC (1990). Official Methods of Analysis of the Association Official Analytical Chemists. $15^{\text {th }}$ Ed. Pub. by the Ass. Offic. Analyt. Chem., Inc. Anlington, Virginia 22201, USA.

Abad, M., P. Noguera and S. Bures (2001). National inventory of organic wastes for use as growing media for ornamental potted plant production: case study in Spain. Bioresource Technol., 77: 197-200.

AboSedera, F.A., N.S. Shafshak, A.S. Shams, M.A. Abul-Soud and M.H. Mohammed (2015). The utilize of vermicomposting outputs in substrate culture for producing snap bean. Ann. Agric. Sci., Moshtohor, 53 (2): 139-151.

Abul-Soud, M.A., M.S.A. Emam, M.A.A. Abdrabbo, F.A. Hashem and S.H. AbdElrahman (2014). Sustainable urban horticulture of sweet pepper via vermicomposting in summer season. J. Adv. in Agric., 3 (1) : 110-122.

Abul-Soud, M.A., M.S.A. Emam and N.G. Abd El-Rahman (2015). The potential use of vermicompost in soilless culture for producing strawberry. Int. J. Pl. and Soil Sci., 8 (5): 1-15.

Alnayef, M. (2012). Understanding the physiological, biochemical, and molecular mechanisms of salinity tolerance in strawberry cultivars and in HvTPK1- Over expressed Barley. Ph.D. Thesis, Università di Bologna, 115.

Ameri, A., A. Tehranifar, G.H. Davarynejad and M. Shoor (2012a). Effect of substrate and cultivar in quality of strawberry. J. Biol. and Environ. Sci., 6 (17): 181-188.

Ameri, A., A. Tehranifar, M. Shoor and G.H. Davarynejad (2012b). Effect of substrate and cultivar on growth characteristic of strawberry in soilless culture system. Afr. J. Biotechnol., 11 (56): 11960-11966.

Arancon, N.Q., C.A. Edwards, R. Dick and L. Dick (2007). Vermicompost tea production and plant growth impacts. Biocycle advancing composting, organics recycling and renewable energy. 419 state avenue, Emmaus, PA 18049-3097. 610-967-4135. www.biocycle.net.

Baldwin, K.R. and J.T. Greenfield (2000). Composting on Organic Farms. North Carolina Cooperative Extension Service, 20 P. Online: http://cefs.css.ncsu.edu.

Benito, M., A. Masaguer, R. De Antonio and A. Moliner (2005). Use of pruning waste compost as a component in soilless growing media. Bioresource Technol., 96: 597-603.

Bidaki, S., V. Chalavi and H. Pirdashty (2013). Effect of vermicompost, cattle manure and different fertilization timing in soilless culture on yield and yield components of strawberry (Fragaria $\times$ ananassa Duch). J. Sci. and Technol. Greenhouse Cult., 4, Isfahan Univ. Technol., Isf., Iran.

Bohlin, C. and P. Holmberg (2004). Peat dominating growing medium in Swedish horticulture. Acta Hort., 644: 177-181.

Carpenter, J., L. Gianessi and L. Lynch (2000). The Economic Impact of the Scheduled U.S. Phaseout of Methyl Bromide. Nat. Cent. Food and Agric. Policy, Washington, DC.

Celikel, G. (1999). Effect of different substrates on yield and quality of tomato. Acta Hort., 486: 353357.

Chapman, H.D. and F.P. Pratt (1961). Ammonium vandate-molybdate method for determination of phosphorus. In: Methods of analysis for soils, plants and water. $1^{\text {st }} \mathrm{Ed}$. California: California Univ., Agric. Division, 184-203.

Cramer, G.R., E. Epstein and A. Luchli (1991). Effects of sodium, potassium and calcium on salt-stressed barley. 2. Elemental analysis. Physiol. Plantarum, 81, 197-202.

Crespo, P. (2010). Variability of health and taste promoting compounds in strawberry (Fragraria $x$ ananassa) fruits. Zurich. ecollection.library.ethz.ch/eserv/eth:2201/eth2201-2202.

Dominguez, J., C.A. Edwards and S. Sulber (1997). A comparison of vermicomposting and composting methods to process animal wastes. Biocycle, 38: 57-59. 
Domínguez, J. (2004). State of the Art and New Perspectives on Vermicomposting Research. In: Edwards, C. (Ed.), Earthworm Ecol. (2 ${ }^{\text {nd }}$ Ed.). CRC Press, Boca Raton, FL, 401-424.

Ebrahimi, R., F. Ebrahimi and M. Ahmadizadeh (2012). Effect of different substrates on herbaceous pigments and chlorophyll amount of strawberry in hydroponic cultivation system. Am. Eurasian J. Agric. and Environ. Sci., 12 (2): 154-158.

El-Behairy, U.A. (1994). The effect of levels of phosphorus and zinc in the nutrient solution on macro and micronutrients uptake and translocation in cucumber (Cucumus sativus L.) grown by nutrient film technique. Ph.D. Thesis, London Univ., 299.

Ercisli, S., U. Sahin, A. Esitken and O. Anapali (2005). Effects of some growing media on the growth of strawberry cvs. "Camarosa" and "Fern". Acta Agrobotanica, 58 : 185-191.

Fascella, G. (2015). Growing substrates alternative to peat for ornamentalplants. Soilless Culture - Use of Substrates for the Production of Quality Horticultural Crops. 47-67. Ed: Md. Asaduzzaman, ISBN 978-953-51-1739-1.

Firoozabadi, M., A. Amrolahi and H. Hokmabadi (2009). Strawberry (F.selva) Effect of different concentration of nitrogen, calcium, potassium in growing. Sixth Iranian Cong. Hort. Sci., Guilan, Iran.

Ghehsareh, A.M. and N. Samadi (2012). Effect of soil acidification on growth indices and microelements uptake by greenhouse cucumber. Afr. J. Agric. Res., 7: 1659-1665

Grattan, S.R. and C.M. Grieve (1999). Mineral nutrient acquisition and response of plants grown in saline environments. In: Handbook of Plant and Crop Stress, M. Pessarakli, (Ed.), Marcel Dekker Press Inc., New York, 203-229, ISBN 0824789873.

Greenway, H. and R. Munns (1980). Mechanisms of salt tolerance in non halophytes. Ann. Rev. Pl. Physiol., 31: 149-190.

Gutiérrez-Miceli, F.A., R.C. García-Gómez, R.R. Rosales, M. Abud-Archila, O.L.M. Angela, M.J.G. Cruz and L. Dendooven. (2008). Formulation of a liquid fertilizer for sorghum (Sorghum bicolor (L.). Moench) using vermicompost leachate. Bioresource Technol., 99: 6174-6180.

Hargreaves, J.C., M.S. Adl and P.R. Warman (2009). Are compost teas an effective nutrient amendment in the cultivation of strawberries? Soil and plant tissue effects. J. Sci. Food Agric., 89: 390-397

Hashemimajd, K., M. Kalbasi, A. Golchin and H. Shariatmadari (2004). Comparison of vermicompost and composts as potting media for growth of tomatoes, J. Pl. Nut., 27: 1107-1123.

He, X., S.J. Traina and T.J. Logan (1992). Chemical properties of municipal solid waste composts. J. Environ., Quality, 21: 318-329.

Herms, D.A. and W.J. Mattson (1992). The dilemma of plants: To grow or defend. The Quarterly Rev. Biol., 67 : 283-335.

Hesami, A., S.S. Khorami, F. Amini and A.B. Kashkooli (2012). Date-peat as an alternative in hydroponic strawberry production. Afr. J. Agric. Res., 7 (23): 3453 - 3458.

Inbar, Y., Y. Hadar and Y. Chen (1993). Recycling of cattle manure: The composting process and characterization of maturity. J. Environ. Quality, 22: 857-863.

Ingham, E.R. (2005). The compost tea brewing manual., $5^{\text {th }}$ Ed. Soil Foodweb Incorporated, Corvallis, Oregon, 79.

Jafarnia, S., A. Hatamzadeh and A. Tehranifar (2010). Effect of different substrates and varieties on yield and quality of strawberry in soilless culture. Adv. in Environ. Biol., 4 (2): 325-328.

Jarecki, M.K., C. Chong and R.P. Vroney (2005). Evaluation of compost leachates for plant growth in hydroponic culture. J. Pl. Nut., 28: 651-667.

Jensen, N.L. (2011). Physiological adaptation and fruit quality of Fragaria $\times$ ananassa under different deficit irrigation strategies and salt treatments. Ph.D. Thesis, Copenhagen Univ., 100.

Keutgen, A.J. and E. Pawelzik (2007). Modifications of taste-relavant compounds in 
strawberry fruit under $\mathrm{NaCl}$ salinity. Food Chem.,105: 1487-1494.

Keutgen, A.J. and E. Pawelzik (2008). Quality and nutritional value of strawberry fruit under long term salt stress. Food Chem., 107: 1413-1420.

Lazcano, C., J. Arnold, A. Tato, J.G. Zaller and J. Domínguez (2009). Compost and vermicompost as nursery pot components: effects on tomato plant growth and morphology. Spanish J. Agric. Res., 7 (4): 944-951.

Lazcano, C. and J. Domínguez (2010). Effects of vermicompost as a potting amendment of two comercially-grown ornamental plant species. Spanish J. Agric. Res., 8 (4): 12601270.

Litterick, A.M., L. Harrier, P. Wallace, C.A. Watson and M. Wood (2004). The role of uncomposted materials, composts, manures and compost extracts in reducing pest and disease incidence and severity in sustainable temperate agricultural and horticultural crop production - A review. Critical Rev. in Pl. Sci., 23 (6):453-479.

Mahmoud, A.M.A., M.M.I. Afifi and M.A. ElHelaly (2014). Production of organic tomato transplants by using Compost as alternative substrate for peat-moss., American-Eurasian J. Agric. and Environ. Sci., 14 (10): 10951104.

Maksimovic, I. and Ž. Ilin (2002). Effects of Salinity on Vegetable Growth and Nutrients Uptake. In: Irrigation Systems and Practices in Challenging Environ., Ed., Lee, TS., 169190. www.intechopen.com.

Márquez-Quiroz, C., S. López-Espinosa, E. Sánchez-Chávez, M. García-Bañuelos, D. la Cruz-Lázaro and J. Reyes-Carrillo (2014). Effect of vermicompost tea on yield and nitrate reductase enzyme activity in saladette tomato. J. Soil Sci. and Pl. Nut., 14: 223-231.

Mazuela, P., M. Urrestarazu and E. Bastias (2012). Vegetable Waste Compost Used as Substrate in Soilless Culture, Crop Production Technologies, Dr. Peeyush Sharma (Ed.). In: Tech, Available from http:// www. intechopen. com / books / crop- productiontechnologies / vegetablewaste compost- used-assubstratein-soilless-culture.

Mitcham, E.J., C.H. Crisosto and A.A. Kader (2000). Strawberry: Recommendations for maintaining postharvest quality. Calif. Univ., Davis.

NOSB (2004). National Organic Standards Board, Compost Tea Task Force Report. Available: http://www. ams. usda. gov/nosb/ meetings/Compost Tea Task Force Final Report. pdf [24 August 2007].

Pant, A.P. (2009). Vermicompost extracts influence growth, mineral nutrients, phytonutrients and antioxidant activity in pakchoi (Brassica rapa cv. Bonsai, Chinesis group) grown under vermicompost and chemical fertilizer. J. Sci. and Food Agric., 89: 2383-2392.

Pant, A.P., T.J.K. Radovic, A.V. Hue and N.Q. Aroncon (2009). Effect of vermicompost tea on pakchoi yield, quality and soil biological proprieties. Comp. Sci. and Utiliz., 19 : 279292.

Parida, A.K. and A.B. Das (2005) Salt tolerance and salinity effects on plants: a review. Ecotoxicol. and Environ. Saf., 60: 324-349.

Pinamonti, F., G. Stringari, F. Gasperi and G. Zorzi (1997). The use of compost: its effects on heavy metal levels in soil and plants. Resouces, Conserv. and Recycling, 21, 129143.

Pirlak, L. and A. Esitken (2004) Salinity effects on growth, proline and ion accumulation in strawberry plants. Acta Agric. Scandinavica. Section B - Plant Soil Sci., 54, 189-192.

Por-Hossein, L., A. Ebadi and Y. Mostufi (2009). Effect of nutrient solution's EC levels and type of media on strawberry growth and yield in hydroponic system. $6^{\text {th }}$ Iranian Cong. of Hort. Sci., Guilan, Iran

Raul, I.C. (1996). Meassuring physical properties. Rutgers Cooperative Extension. New Jersey Agric. Exp. Station. New Jersey Univ.

Raviv, M. and S. Medina (1997). Physical characteristics of separated cattle manure compost. Comp. Sci. and Util., 5 (3): 44-47.

Robertson, RA. (1993). Peat, horticulture and environment. Biodivers. Conserv., 2 : 541-547. 
Roberts, P., D.L. Jones and G. Edwards-Jones (2007). Yield and vitamin C content of tomatoes grown in vermicomposted wastes. J. Sci. of Food and Agric., 87:1957-1963.

Rodda, M.R.C., L.P. Canellas, A.R Façanha, D.B. Zandonadi, J.G.M. Guerra, D.L. De Almeida, and G.A. De Santos (2006). Improving lettuce seedling root growth and ATP hydrolysis with humates from Vermicompost. II- Effect of Vermicompost source. RevistaBrasileira de Ciencia do Solo, 30: 657-664.

Saied, A.S., A.J. Keutgen and G. Noga (2005). The influence of $\mathrm{NaCl}$ salinity on growth, yield and fruit quality of strawberry cvs. 'Elsanta' and 'Korona'. Scientia Hort., 103 : 289-303.

Santiago-López, G., P. Preciado-Rangel, E. Sánchez-Chavez, J.R. Esparza-Rivera, M.
Fortis-Hernández and A. Moreno-Reséndez (2016). Organic nutrient solutions in production and antioxidant capacity of cucumber fruits. Em. J. Food and Agric., 28 (7): 518-521.

Shannon, M.C. and C.M. Grieve (1999). Tolerance of vegetable crops to salinity. Scientia Hort., 78: 5-38.

Snedecor, G.W. and W.G. Cochran. (1981). "Statistical Methods" $7^{\text {th }}$ Ed., Iowa State Univ., Press, Ames, Iowa, USA, 225-330.

Welke, S. (2005). The effect of compost extract on the yield of strawberries and the severity of Botrytis cinerea. J. Sustainable Agric., 25 (1): 57-68.

Wilson, G.C.S (1983). The physico-chemical and physical properties of horticultural substrate. Acta Hort., 150 : 19-32.

\title{
تأثير البيئات والمحاليل المغذية المختلفة علي النمو الخضري والمحتوى الكيمائي والإنتاج وجودة ثمار الفراولة النمو
}

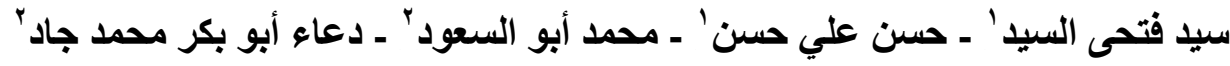

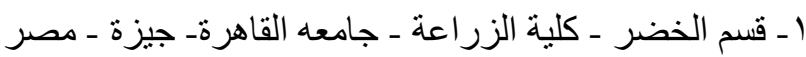

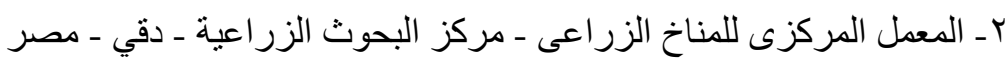

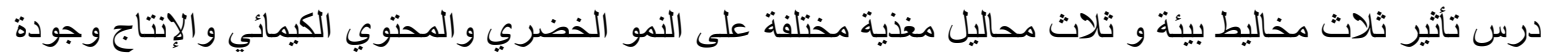

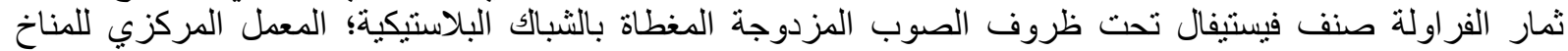

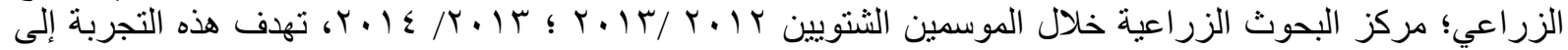

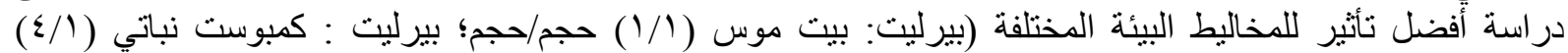

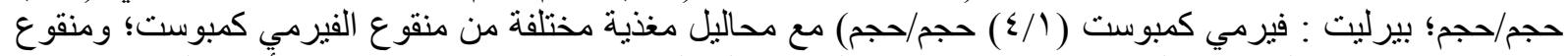

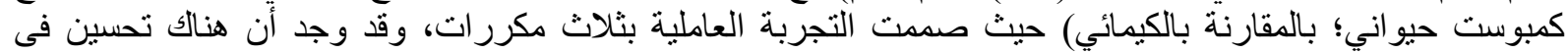

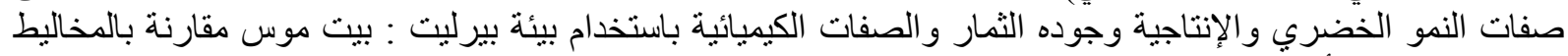

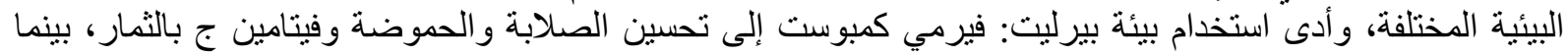

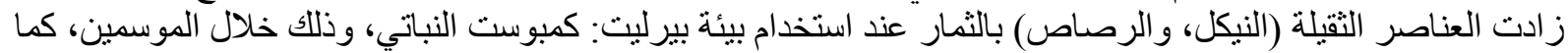

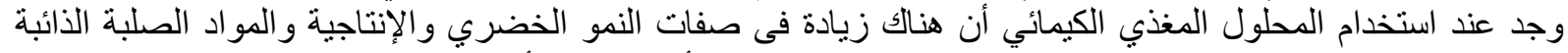

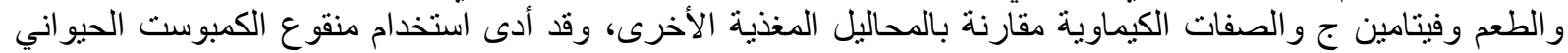

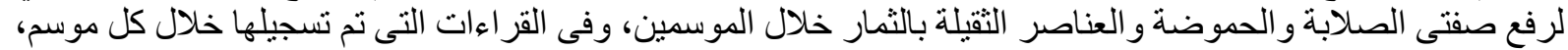

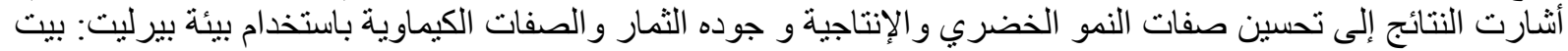

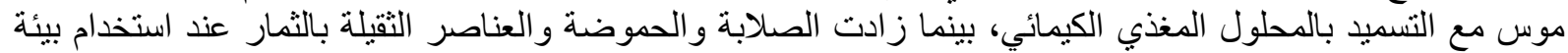
بيرليت : فيرمي كمبوست مع التسميد بمنقوع كمبوست الني حيو اني خلال الموسمين. 